\title{
Intramural gas in the small bowel followed by chronic obstruction
}

\author{
R.P. Waldron, D. Dawkins and I.A. Donovan \\ Department of Surgery, Dudley Road Hospital, Dudley Road, Birmingham B18 7QH, UK.
}

\begin{abstract}
Summary: A 63 year old male presented with recurring abdominal pain dating from an acute episode 3 months previously. Abdominal X-rays in the acute phase demonstrated small intestinal intramural gas. A jejunal stricture with panmural necrosis was resected.
\end{abstract}

\section{Introduction}

The radiological demonstration of intestinal intramural gas in an adult was first documented by KayButler (1962). The value of early recognition of this roentgenographic sign cannot be over-emphasized, as it usually indicates extensive panmural intestinal necrosis (Berne \& Halls, 1983). In this paper we report a case in which this sign was missed at the time of the acute pain, but when identified it proved useful in the patient's management.

\section{Case report}

A 63 year old male presented with acute upper abdominal pain of $12 \mathrm{~h}$ duration, associated with vomiting and borborygmi. He had sustained a myocardial infarct $5 \mathrm{y}$ previously and was known to have peripheral vascular insufficiency with no palpable pulsation below the femorals on either side. On examination, the abdomen was tender with guarding in the left upper quadrant. Bowel sounds were within normal limits. This episode settled on conservative management. He presented again 3 months later with recurrent episodes of colicky abdominal pain, aggravated by eating and associated with vomiting, borborygmi and abdominal distension. Abdominal examination did not disclose any abnormality. Abdominal X-rays demonstrated dilated proximal small bowel loops. Review of the plain abdominal Xrays taken 3 months previously revealed the presence of intramural gas in a loop of jejunum (Figure 1). Barium follow-through confirmed a dilated segment of proximal jejunum with a short stricture distal to it. Arteriography demonstrated an atheromatous

Correspondence: R.P. Waldron M.Ch., F.R.C.S.

Accepted: 9 October 1984 abdominal aorta, but no abnormality of the coeliac or mesenteric vessels. Laparotomy confirmed a localized stricture of the proximal jejunum which was treated by local resection. An area of full thickness ischaemic necrosis was confirmed histologically. Post-operative recovery was uneventful.

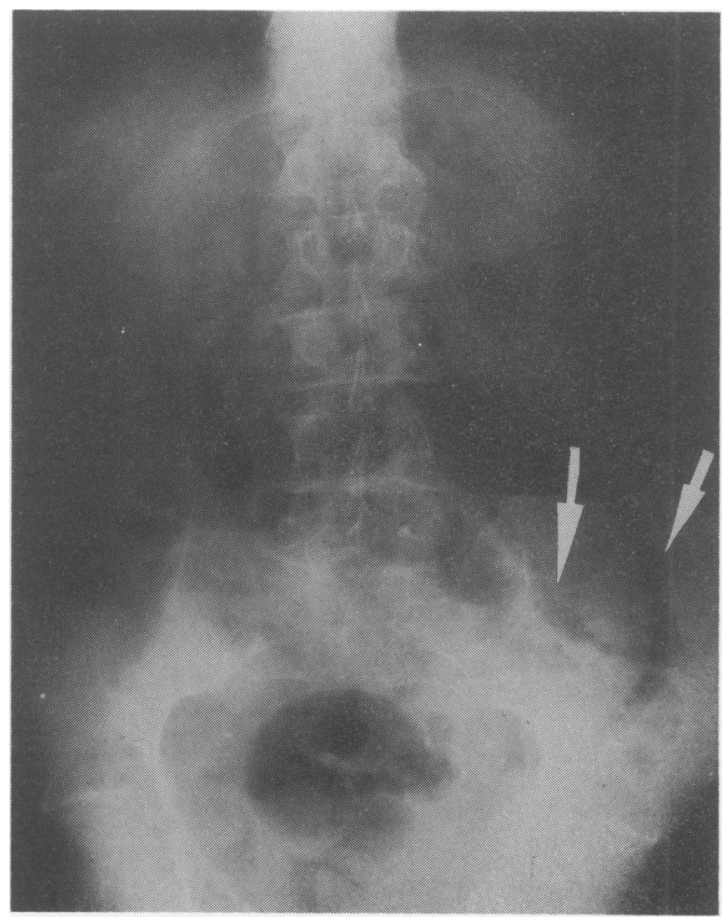

Figure 1 Plain abdominal X-ray revealing the presence of intramural gas in a loop of jejunum.

(C) The Fellowship of Postgraduate Medicine, 1985 


\section{Discussion}

Ischaemic damage to the small bowel mucosa permits the entry of gas-forming organisms into the intestinal wall with consequent interstitial emphysema (Lynn \& Hodes, 1973). The increase in intestinal luminal pressure may force air through the damaged mucosa. The presence of intramural gas on a plain abdominal film in the presence of abdominal pain is an important radiological sign indicative of underlying intestinal wall necrosis making early surgical intervention desirable as free perforation often follows. In this case the necrotic area became walled off by adjacent loops of bowel. Even if the process heals by fibrosis a resection of the stricture will anyway be necessary.

\section{References}

BERNE, T.V. \& HALLS, M.H. (1983). Intramural gas in adults. Surgery, Gynecology and Obstetrics, 159, 479.

KAY-BUTLER, J.J. (1962). Intersitial emphysema of the caecum. Gut, 3, 267.

LYNN, W. \& HODES, P.J. (1973). A new sign of small bowel volvulus. Radiology, 108, 289. 